\title{
The Correlation Analysis Between the Blood Lipids, Glycosylated Protein Level and Inflammatory Factors of Pregnant Women with GDM During Pregnancy and the Weight of Newborn
}

\section{Changhua Hu}

Hanyang Hospital, Wuhan University of Science and Technology $₫$ Wuhan 430053هHubei province Lirong Wu ( $\sim$ Lirong_wu163@hotmail.com )

Hanyang Hospital, Wuhan University of Science and Technology $₫$ Wuhan 430053囚Hubei province Juan Fang

Hanyang Hospital, Wuhan University of Science and Technology 『Wuhan 430053هHubei province

\section{Research Article}

Keywords: Diabetes during pregnancy, blood lipid, glycosylated protein, inflammatory factors, the weight of newborn

Posted Date: September 29th, 2021

DOl: https://doi.org/10.21203/rs.3.rs-896430/v1

License: (c) (1) This work is licensed under a Creative Commons Attribution 4.0 International License.

Read Full License 


\section{Abstract}

The study was carried to explore the correlation between blood lipids, blood glucose levels, inflammatory factor and weight of newborn, to provide reference for control of blood glucose in pregnant women with gestational diabetes mellitus (GDM) and early screening of macrosomia. Fifty pregnant women (give birth to newborn) with GDM were selected as research group, and 55 normal pregnant women (give birth to newborn) as control group. Blood lipid levels of TC, TG, HDL-C, LDL-C, the fasting blood glucose (FPG), $\mathrm{HbAlc}$, glycosylated albumin (GA), and expression of inflammatory factor TLR4 of pregnant women in the two groups were monitored. The levels of TG, FPG, HbA1c and GA in pregnant women, the levels of TLR4 in cord blood of newborn, the relative expression of TLR4 protein and TLR4mRNA in the placenta were higher than in control group, and level of HDL-C was lower than the control group. The levels of TC and LDL-C of pregnant women were higher than in control group. Weight of newborn was positively correlated with these all except HDL-C levels (negatively correlated) and no correlation was found with TC and LDLC. The weight of newborn and incidence of macrosomia in research group were higher compared to control group, and scores of newborns at $1 \mathrm{~min}$ and $5 \mathrm{~min}$ were lower compared to control group. The results revealed that strengthening the detection of blood lipid and blood glucose during pregnancy can prevent adverse outcomes such as giant babies and improve the quality of birth.

\section{Introduction}

A series of changes in the secretion system and hormone levels of pregnant women during pregnancy will trigger changes in glucose and lipid metabolism [1]. The first occurrence or discovery of abnormal glucose metabolism during pregnancy is called Gestational Diabetes Mellitus (GDM), and its onset is related to insulin resistance and decreased secretion of islet cells [2-3]. Pregnant women with GDM during pregnancy are prone to adverse pregnancy outcomes, which increase the risk of huge babies, neonatal hypoglycemia, deformity, asphyxia and even death. And pregnant women with GDM during pregnancy have a high probability of developing T2DM [4]. Therefore, GDM is more harmful to mothers and babies.

With the development of the economy, the living standards have improved significantly, and the gestational age of women has gradually been pushed back. Many pregnant women have supplied excessive nutrition during pregnancy, lack of necessary exercise and other factors, resulting in an increasing trend in the incidence of GDM year by year [5]. Early diagnosis of GDM patients and early intervention are of great significance for reducing complications of GDM pregnant women and newborns, and improving adverse pregnancy outcomes.

Disorders of glucose and lipid metabolism are the key to GDM research. GDM pregnant women have higher levels of VLDL, TC, and TG in blood circulation [6]. When pregnant women are diagnosed, they mainly rely on measuring blood glucose at fasting and 2 hours after meal to guide pregnant women to control blood glucose during pregnancy, but the stability of immediate blood glucose is low and blood glucose control is difficult to standardize [7]. The detection of the levels of glycosylated hemoglobin and 
glycosylated albumin does not require fasting, and it has a good stability. Therefore, it is more suitable as an indicator for the detection of blood glucose in pregnant women with GDM [8-9]. In recent years, domestic and foreign studies have shown that chronic aseptic inflammatory response is also closely related to GDM. When pancreatic islet cell function is impaired, insulin resistance is aggravated, and insulin resistance is one of the recognized pathogenic factors of GDM [10].

Based on a large number of domestic and foreign literatures, this study collected and analyzed the correlation between the levels of blood lipid, glycosylated protein and inflammatory factors of GMD pregnant women and the weight of newborns, and provided theoretical basis for the control of blood glucose in pregnant women with GMD and early screening of macrosomia. The detailed descriptions are demonstrated as follows:

\section{Materials And Methods}

\subsection{General information}

50 GDM pregnant women and their newborns who were hospitalized in the obstetrics department of our hospital from August 2020 to January 2021 were selected as the research group, and 55 normal pregnant women and their newborns who were hospitalized during the same period were selected as the control group.

Considering IADPSG as the diagnostic criteria for pregnant women with GDM, 75g OGTT was performed to screen whether pregnant women are GDM at the first visit between 24-28 weeks of pregnancy [11]. When the pregnant woman's fasting blood glucose is $\geq 5.1 \mathrm{mmol} / \mathrm{L}$ or 1 -hour blood glucose $\geq 10 \mathrm{mmol} / \mathrm{L}$ or 2 hours' blood glucose $\geq 8.5 \mathrm{mmol} / \mathrm{L}, \mathrm{GDM}$ can be diagnosed when any one of the above is met.

Inclusion criteria: $₫ 20-35$ years old; $\nabla$ Natural conception; $\nabla$ Diagnosed with GDM at 24-28 weeks of gestation; $\varangle$ No abnormalities such as Down syndrome, malformation, etc. found on various screenings during pregnancy; $\varangle$ Follow-up examinations will be performed every 4 weeks after diagnosis until delivery.

Exclusion criteria: $₫$ Diabetes complicated with pregnancy; $₫$ Congenital or genetic diseases; $\varangle$ Multiple pregnancy; $\otimes$ Pregnant women with other chronic diseases during pregnancy.

According to the situation of GDM, 50 GDM pregnant women and their newborns were taken as the research group, and 55 normal pregnant women who were hospitalized during the same period were taken as the control group. The general clinical data of age, gestational week, gestation times and prepregnancy weight in two groups were not significantly different $(P>0.05)$, and they were comparable.

On the basis of obtaining the consent of the pregnant woman and their family members, the informed consent form is signed, and they voluntarily joined the research project. Then we collect relevant 
information of pregnant women, and the information comes from various clinical examination index values. This study has been reviewed and approved by the ethics committee of our hospital.

\subsection{Methods}

\subsubsection{Determination of blood lipid}

After 12 hours of fasting, the pregnant women of the two groups pumped $10 \mathrm{ml}$ of cubital venous blood in the morning. After the blood was drawn, the upper serum was obtained after anticoagulation and centrifugation. Cobas-8000 automatic biochemical analyzer (Roche, Germany) was used to determine the TC, TG, HDL-C, and LDL-C in serum.

\subsubsection{Determination of blood glucose}

After 12 hours of fasting, the pregnant women of the two groups pumped $10 \mathrm{ml}$ of cubital venous blood in the morning. After the blood was drawn, the upper serum was obtained after anticoagulation and centrifugation. MQ-600 glycosylated hemoglobin analyzer (Nanjing Hanyu Medical Technology Co., Ltd.) was used to determine the levels of glycosylated hemoglobin (HbAlc) and glycosylated albumin (GA).

\subsubsection{Determination of inflammatory factors}

After the umbilical cord was broken at birth, $5 \mathrm{ml}$ of cord venous blood was drawn from the newborn. After centrifugation, the upper serum was placed in a cryotube and stored in a refrigerator at $-20^{\circ} \mathrm{C}$. Enzymelinked immunoassay (ELISA) was used to detect the expression level of TRL4, operate according to the instructions of the kit (Wuhan Huamei Biological Co., Ltd.), repeat twice and take the average value.

The imprinting method of Western Blot was used to detect the protein level of TLR4 in the placenta.

RT-PCR method was used, RNA purification and amplification was performed through extraction, separation, precipitation, washing, the mRNA expression level of TLR4 in the placenta was detected.

Observation Indicators and Evaluation Standards

\subsubsection{The level of Blood Lipid}

Clinically, TC, TG, HDL-C, LDL-C indicators are used to reflect the blood lipid levels of pregnant women. Diabetes during pregnancy can cause fat metabolism disorders, which in turn triggers changes in the levels of TC, TG, HDL-C, and LDL-C [12].

\subsubsection{The Level of Blood Glucose}

Clinically, the fasting blood glucose (FPG) index is used to reflect the blood glucose level of pregnant women when measured. FPG is the micro-control index of blood glucose.

$\mathrm{HbAlc}$ is a ketamine compound formed by the condensation of hemoglobin and glucose in the blood. The condensation process is slow and irreversible. The level of HbAlc is related to the degree of glycosylation. 
The measurement of this indicator can reflect the average blood glucose level of pregnant women 2-3 months before measurement [13].

GA is a kind of ketamine compound formed by the non-enzymatic reaction between the 4 Lysines of glucose and the N-terminus of serum albumin. And the structure of GA is relatively stable. The half-life period of GA is the same as that of albumin, which can reflect the overall level of serum FPG. The measurement of this indicator can reflect the average blood glucose level of pregnant women 2-3 weeks before the measurement [14].

The three indicators reflect at different times and have their own characteristics and clinical significance. Thus, the combined detection of the three indicators was used in this paper during pregnancy to reflect the recent and full blood glucose level of the pregnant woman.

\subsubsection{The Level of Inflammatory Factor TLR}

TLR4 is the most important receptor in the TLR family. The enhancement of maternal immune system mediated by TLR4 during pregnancy will aggravate insulin resistance [15-16].

\subsubsection{The Apgar Score of Newborn}

The five physical signs of newborn (heartbeat, breathing, muscle tension, stress response and skin color) is analyzed at 1 and 5 minutes after delivery based on the Apgar score sheet. Each item is scored 0-2 points, and the total score is 10 points. The status of the newborn is determined according to the total score. The higher the score is, the better the state of newborn is [17].

\section{Results}

\subsection{Comparison in the general clinical indicators of pregnant women between the two groups}

Through comparison, it is found that the difference in general clinical indicators such as age, gestational week, and gestational times of two groups are not statistically significant $(P>0.05)$, and they are comparable (Table 1). 
Table 1

Comparison in the general clinical indicators of pregnant women between the two groups $(\bar{x} \pm s) /[n$ (\%) ]>

\begin{tabular}{|lllll|}
\hline general clinical data & $\begin{array}{l}\text { the control group }(\mathrm{n}= \\
\mathbf{5 5})\end{array}$ & $\begin{array}{l}\text { the research group }(\mathrm{n}= \\
\mathbf{5 0})\end{array}$ & $\boldsymbol{t} / X^{2}$ & $\boldsymbol{P}$ \\
\hline age(year) & $29.62 \pm 2.83$ & $30.02 \pm 2.09$ & 1.10 & 0.272 \\
\hline gestational age(week) & $39.81 \pm 1.73$ & $39.48 \pm 1.82$ & 1.41 & 0.160 \\
\hline gravidity(time) & $1.73 \pm 0.77$ & $1.92 \pm 0.83$ & 0.85 & 0.395 \\
\hline weight before pregnancy(year) & $61.54 \pm 11.62$ & $59.10 \pm 9.35$ & 1.32 & 0.187 \\
\hline $\begin{array}{l}\text { weight gain during } \\
\text { pregnancy(kg) }\end{array}$ & $15.64 \pm 4.32$ & $16.07 \pm 3.87$ & 0.71 & 0.459 \\
\hline
\end{tabular}

\subsection{Comparison in the blood lipid levels of pregnant women between the two groups}

The TG level of pregnant women in the research group is $3.30 \pm 1.09 \mathrm{mmol} / \mathrm{L}$, which was significantly higher than the TG level $(2.79 \pm 1.07) \mathrm{mmol} / \mathrm{L}$ of pregnant women in the control group, and the level of $\mathrm{HDL}-\mathrm{C}(1.72 \pm 0.46) \mathrm{mmol} / \mathrm{L}$ was significantly lower than that in the control group $(2.10 \pm 0.42) \mathrm{mmol} / \mathrm{L}$, and the differences in the levels of TG and HDL-C of pregnant women between the two groups were statistically significant $(P<0.05)$. The level of TC $(6.18 \pm 1.20) \mathrm{mmol} / \mathrm{L}$ and the level of LDL-C $(3.30 \pm$ $1.01) \mathrm{mmol} / \mathrm{L}$ in the research group were slightly higher than those in the control group (5.82 \pm 1.14$)$ $\mathrm{mmol} / \mathrm{L}$ and $(3.14 \pm 0.81) \mathrm{mmol} / \mathrm{L}$, respectively, But the difference between the two groups was not statistically significant $(P>0.05)$ (Fig. 1). The weight of newborn was positively correlated with the TG level of pregnant women in the research group, and negatively correlated with the HDL-C level. However, no correlation was found with TC and LDL-C levels (Table 2).

Table 2

Correlation analysis between the level of blood lipid in pregnant women and the weight of newborn in the research group

\begin{tabular}{|lllllllll|}
\hline index & TG & \multicolumn{3}{c}{ TC } & \multicolumn{3}{c|}{ HDL-C } & \multicolumn{2}{l|}{ LDL-C } \\
\cline { 2 - 9 } & $\boldsymbol{r}$ & $\boldsymbol{P}$ & $\boldsymbol{r}$ & $\boldsymbol{P}$ & $\boldsymbol{r}$ & $\boldsymbol{P}$ & $\boldsymbol{r}$ & $\boldsymbol{P}$ \\
\hline weight of newborn & 0.817 & 0.001 & 0.153 & 0.294 & -0.682 & 0.001 & 0.120 & 0.419 \\
\hline
\end{tabular}

\subsection{Comparison in the blood glucose levels of pregnant women between the two groups}

The FPG level $(4.82 \pm 1.54) \mathrm{mmol} / \mathrm{L}, \mathrm{HbA} 1 \mathrm{c}$ level $(7.54 \pm 1.83) \%$ and GA level $(3.52 \pm 1.05)$ of pregnant women in the research group were significantly higher than those in the control group $(4.16 \pm 1.10)$ $\mathrm{mmol} / \mathrm{L},(4.95 \pm 2.17) \%$ and $(2.14 \pm 0.97) \mathrm{mmol} / \mathrm{L}$, and the differences were statistically significant $(P<$ 
0.05) (Fig. 2). The weight of newborns was positively correlated with the levels of FPG, HbA1c and GA in the cord blood of pregnant women in the research group (Table 3).

Table 3

Correlation analysis between the level of blood glucose in pregnant women and the weight of newborn in the research group

\begin{tabular}{|lllllll|}
\hline index & FPG & \multicolumn{3}{c|}{ HbA1c } & \multicolumn{3}{l|}{ GA } \\
\cline { 2 - 7 } & $\boldsymbol{r}$ & $\boldsymbol{P}$ & $\boldsymbol{r}$ & $\boldsymbol{P}$ & $\boldsymbol{r}$ & $\boldsymbol{P}$ \\
\hline weight of newborn & 0.785 & 0.001 & 0.821 & 0.000 & 0.617 & 0.002 \\
\hline
\end{tabular}

\subsection{Comparison in the TLR4 levels of pregnant women between the two groups}

The level of TLR4 (4193.02 \pm 799.53$) \mathrm{pg} / \mathrm{mL}$ in the umbilical cord blood of newborn in the research group was significantly higher than that in the control group $(3669.26 \pm 769.62) \mathrm{pg} / \mathrm{mL}$. The relative expression of TLR4 protein and TLR4mRNA in the placenta of the pregnant women in the research group were respectively significant higher than those in the control group, and the differences were statistically significant $(P<0.05)$ (Fig. 3). The weight of the newborn was positively correlated with the level of TLR4 in the cord blood of the pregnant women, the relative expression of TLR4 protein in the placenta and TLR4mRNA (Table 4) in the research group.

Table 4

Correlation analysis between the level of TLR4 in pregnant women and weight of newborn in research group

\begin{tabular}{|lllllll|}
\hline index & TLR4 & \multicolumn{3}{c|}{ TLR4 protein } & \multicolumn{2}{l|}{ LR4mRNA } \\
\cline { 2 - 7 } & $\boldsymbol{r}$ & $\boldsymbol{P}$ & $\boldsymbol{r}$ & $\boldsymbol{P}$ & $\boldsymbol{r}$ & $\boldsymbol{P}$ \\
\hline weight of newborn & 0.892 & 0.000 & 0.873 & 0.000 & 0.853 & 0.000 \\
\hline
\end{tabular}

\subsection{Comparison in the situation of newborns between the two groups}

The birth weight of newborns was $(3646.05 \pm 391.97) \mathrm{g}$ and the incidence of macrosomia was $18.00 \%$ in the research group, which were both significantly greater than those in the control group (The birth weight of newborns was $3394.06 \pm 388.26$ and the incidence of macrosomia was $7.27 \%$ ), and the difference was statistically significant $(P<0.05)$. The scores of newborns at $1 \mathrm{~min}$ and $5 \mathrm{~min}$ in the research group were lower than those in the control group, and the difference was statistically significant $(P<0.05)$ (Fig. 4).

\section{Discussion}


Under the combined action of a variety of hormones during pregnancy, the blood lipid level of pregnant women will remain in a high balance during the entire pregnancy, which can not only meet the fetus's needs for nutrients, but also store raw materials for postpartum breastfeeding [18]. The level of TG starts to increase at the 9th week of pregnancy, while TC and LDL-C decrease. At the 12th week of pregnancy, HDL-C and PL continue to increase, especially for pregnant women with GDM [19]. At present, domestic and foreign studies have confirmed that the blood glucose level of pregnant women with GDM will cause the newborn to grow too quickly in the uterus, and even large babies may appear [20]. Research by Hashimoto et al. also showed that there is a linear relationship between GA and adverse pregnancy outcomes [21]. Although the blood glucose level of pregnant women with GDM can be controlled at a reasonable level through drugs, the incidence of macrosomia has not been reduced, which proves that the incidence of macrosomia is related to the blood lipid level [22].

One of the recognized pathogenic factors of GDM is insulin resistance, and chronic inflammation can aggravate insulin resistance [23-24]. Domestic and foreign studies have shown that the expression levels of TLR4, NF-K B and inflammatory factors (such as TNF-a, IL-6 and CRP) in placental tissue or peripheral blood of GDM pregnant women are higher than those in normal pregnant women [25-26]. Giannoukakis et al. used IKB to inhibit the expression of NF-KB and reduce the damage of inflammatory factors to pancreatic $\beta$ cells [27]. These studies prove that TLR4, NF-KB, and inflammatory factors are closely related to GDM.

In this study, the blood lipid levels (TC, TG, HDL-C, LDL-C), the blood glucose levels (FPG, HbAlc, GA), and inflammatory factor TLR4 expression levels in two groups were monitored. The results showed that the levels of TG, FPG, HbA1c, GA in pregnant women, TLR4 in cord blood of newborn, and the relative expression of TLR4 protein and TLR4mRNA in the placenta in the research group were significantly higher than those in the control group $(P<0.05)$, and the level of HDL-C was significantly lower than that in the control group $(P<0.05)$. The weight of newborns was positively correlated with the levels of $T G, F P G$, HbA1c, and GA of pregnant women in the research group, the levels of TLR4 in the umbilical cord blood of pregnant women, and the relative expression of TLR4 protein and TLR4mRNA in the placenta, and negatively correlated with the levels of HDL-C, but there is no correlation with TC and LDL-C was found. The reason may be that the high levels of blood glucose and blood lipid in pregnant women with GDM will directly enter the newborn's body through the umbilical cord, causing the newborn's blood glucose to rise. The stimulation of pancreatic islet cells causes the newborn's hyperinsulinemia. When glucose and fat continue to accumulate in the newborn's body, excessive growth even macrosomia phenomenon will be easy to occur.

\section{Conclusions}

In summary, changes in the levels of blood lipids (TG and HDL-C), blood glucose (FPG, HbA1C and GA) of pregnant women with diabetes during pregnancy will affect the weight of newborns. Therefore, the monitoring of blood lipids and blood glucose of pregnant women with GDM should be strengthened to reduce the risk of mother and child. The innovation of this study is to establish the correlation between 
the levels of blood glucose, blood lipids and TLR4 of pregnant women with GDM and the weight of newborns, expecting to provide a theoretical basis for reducing the adverse pregnancy outcomes of pregnant women with GDM and reasonably controlling the blood glucose and blood lipids during pregnancy. The deficiencies of this study are as follows: (1) The sample included is small, which makes the research results lack of universality. (2) The research is only conducted on the expression level of TLR4, and the results obtained are biased. In view of the above shortcomings, the next step is to expand the sample of GDM pregnant women and reduce the analysis error caused by the limited sample, expecting that more representative and scientific conclusions can be obtained, and reference for improving the pregnancy outcome of GDM pregnant women can be provided.

\section{Abbreviations}

GDM, gestational diabetes mellitus, HDL, high density lipids, LDL, low density lipids, HbAlc, glycosylated hemoglobin. GA, glycosylated albumin, ELISA, Enzyme-linked immunoassay, FGP, fasting blood glucose, TNF-a, tissue necrotic factor-a, IL-6, interleukin-6.

\section{Declarations}

\section{Ethics approval and consent to participate}

All the methods and experiments were carried in accordance to the relevant ethical guidelines and regulations of the Hanyang Hospital Affiliated to Wuhan University of Science and Technology $₫$ Wuhan 430053囚Hubei province, China.

\section{Consent for publication}

"Not Application"

\section{Availability of data and materials}

Data are available upon reasonable request from correspondence.

\section{Competing interests}

All the authors declare that they have no conflict of interest.

\section{Funding}

Authors received no research funding for this study.

\section{Author contributions}

$\mathrm{CH}$ and $\mathrm{LW}$ conceived, designed and supervised the study, interpreted the data, prepared the figure with the related legend, and wrote the manuscript; JF analyzed the data; $\mathrm{CH}$ and JF performed the literature 
search. All authors contributed to editorial changes in the manuscript. All authors read and approved the final manuscript.

\section{Acknowledgments}

"Not Applicable"

\section{References}

1. Celik E,Celik O,Yilmaz E et al.Association of low maternal levels of salusins with gestational diabetes mellitus and with small-for-gestational-age fetuses.[J].Eur J Obstet Gynecol Reprod Biol,2013,1676(1),29-33.

2. Wang XQ,Yang T,Miao JK,et al.Correlation Between Maternal and Fetal Insulin Resistance in Pregnant Women with Gestational Diabetes Mellitus.[J].Clin Lab,2018,64(6):945-953.

3. Kijmanawat A,Panburana P,Reutrakul S,et al.Effects of probiotic supplements on insulin resistance in gestational diabetes mellitus:A double-blind randomized controlled trial.[J].Journal of Diabetes Investigation.2018,10(1),163-170.

4. Szmuilowicz E D,Josefson J L,Metzger B E.Gestational Diabetes Mellitus.[J].Endocrinology and Metabolism Clinics of North America.2019, 48(3),479-493.

5. Chiefari E,Arcidiacono B,Foti D,et al.Gestational diabetes mellitus: an updated overview.[J].Journal of Endocrinological Investigation,2017,40(9), 899-909.

6. Brett, K. E., Ferraro, Z. M., Holcik, M., \& Adamo, K. B.Placenta nutrient transport-related gene expression: the impact of maternal obesity and excessive gestational weight gain.[J].The Journal of Maternal-Fetal \& Neonatal Medicine,2015,29(9), 1399-1405.

7. Huhn $E A$,Linder T,Eppel D,et al.Effectiveness of real-time continuous glucose monitoring to improve glycaemic control and pregnancy outcome in patients with gestational diabetes mellitus: a study protocol for a randomised controlled trial.[J].BMJ Open,2020,10(11), e040498.

8. Jin H.Increased levels of glycosylated hemoglobin, microalbuminuria and serum cystatin $\mathrm{C}$ predict adverse outcomes in high-risk pregnancies with gestational diabetes mellitus.[J].Exp Ther Med,2020,19(2),1281-1287.

9. Yoshiuchi K,Matsuhisa M,Katakami N,et al.Glycated albumi is a better indicator for glucose excursion than glycate hemoglobin in type 1 and type 2 diabetes.[J].Endocr J,2008,55(3),503-507.

10. Świrska J,Zwolak A,Dudzińska M,et al. Gestational diabetes mellitus-literature review on selected cytokines and hormones of confirmed or possible role in its pathogenesis.[J].Ginekol Pol,2018,89(9),522-527.

11. Basri $\mathrm{N}$ I,Mahdy Z A, Ahmad S,et al.The World Health Organization (WHO) versus The International Association of Diabetes and Pregnancy Study Group (IADPSG) diagnostic criteria of gestational diabetes mellitus (GDM) and their associated maternal and neonatal outcomes.[J].Horm Mol Biol Clin Investig,2018,17,34(1). 
12. Herrera E,Desoye G.Maternal and fetal lipid metabolism under normal and gestational diabetic conditions. Hormone Molecular Biology and Clinical Investigation,2016,26(2),109-127.

13. Guo J,Lei S,Zhou Y, et al.The ratio of estimated average glucose to fasting plasma glucose level as an indicator of insulin resistance in young adult diabetes.[J].Medicine,2020,99(40), e22337.

14. Yazdanpanah $S$,Rabiee $M$,Tahriri $M$,et al.Evaluation of glycated albumin (GA) and $G A / H b A 1 c$ ratio for diagnosis of diabetes and glycemic control: A comprehensive review.[J].Critical Reviews in Clinical Laboratory Sciences, 2017,54(4), 219-232.

15. Feng H,Su R,Song Y,et al.Positive Correlation between Enhanced Expression of TLR4/MyD88/NF-k B with Insulin Resistance in Placentae of Gestational Diabetes Mellitus.[J].PLOS ONE,2016,11(6), e0157185.

16. Lyu Y,Jia S,Wang S,et al.Gestational diabetes mellitus affects odontoblastic differentiation of dental papilla cells via Toll-like receptor 4 signaling in offspring.[J].Journal of Cellular Physiology,2019,235(4),3519-3528.

17. Yeagle $K \mathrm{P}, \mathrm{O}^{\prime} B$ rien $\mathrm{J} \mathrm{M}$,Curtin, $\mathrm{W} \mathrm{M}$,et al.Are gestational and type II diabetes mellitus associated with the Apgar scores of full-term neonates?.[J].International Journal of Women's Health,2018,10(8), 603607.

18. Eshriqui I,Franco-Sena A B,Farias D R,et al.Prepregnancy Dietary Patterns Are Associated with Blood Lipid Level Changes During Pregnancy: A Prospective Cohort Study inRio de Janeiro, Brazil. [J].Journal of the Academy of Nutrition and Dietetics,2017, 117(7),1066-1079.

19. Lepsch J,Eshriqui I,Farias $D$ R,et al.Association between early pregnancy vitamin $D$ status and changes in serum lipid profiles throughout pregnancy.[J].Metabolism, 2017,70,85-97.

20. Mack L R,Tomich P G.Gestational Diabetes.[J].Obstetrics and Gynecology Clinics of North America,2017,44(2), 207-217.

21. Hashimoto K,Koga M.Indicators of glycemic control in patients with gestational diabetes mellitus and pregnant women with diabetes mellitus. World Journal of Diabetes,2015,6(8),1045-1056.

22. Hashemipour S,Seidjavadi E H,Maleki F,et al.Maternal triglycerides level is a predictor of fetal macrosomia in non-obese pregnant women with gestational diabetes mellitus.[J].Pediatrics \& Neonatology,2018,59(6),567-572.

23. Hernandez T L,Mande A B,Linda A.Nutrition Therapy Within and Beyond Gestational Diabetes. [J].Diabetes Research and Clinical Practice,2018,145,39-50.

24. Fan G,Jiang X,Wu X,et al.Anti-Inflammatory Activity of Tanshinone IIA in LPS-Stimulated RAW264.7 Macrophages via miRNAs and TLR4-NF-KB Pathway.[J].Inflammation,2016,39(1), 375-384.

25. Kuzmicki M,Telejko B,Wawrusiewicz-Kurylonek N,et al. The expression of genes involved in NF- B activation in peripheral blood mononuclear cells of patients with gestational diabetes.[J].European Journal of Endocrinology,2013,168(3), 419-427.

26. Vitoratos $\mathrm{N}$,Valsamakis $\mathrm{G}$, Mastorakos $\mathrm{G}$,et a.IPre- and early post-partum adiponectin and Interleukin1 beta levels in women with and without gestational diabetes.[J].HORMONES,2008,7(3), 230-236. 
27. Giannoukakis N,Rudert W A,Trucco M,et al.Protection of Human Islets from the Effects of Interleukin$1 \beta$ by Adenoviral Gene Transfer of an IKB Repressor.[J].Journal of Biological Chemistry,2000,275(47), 36509-36513.

\section{Figures}
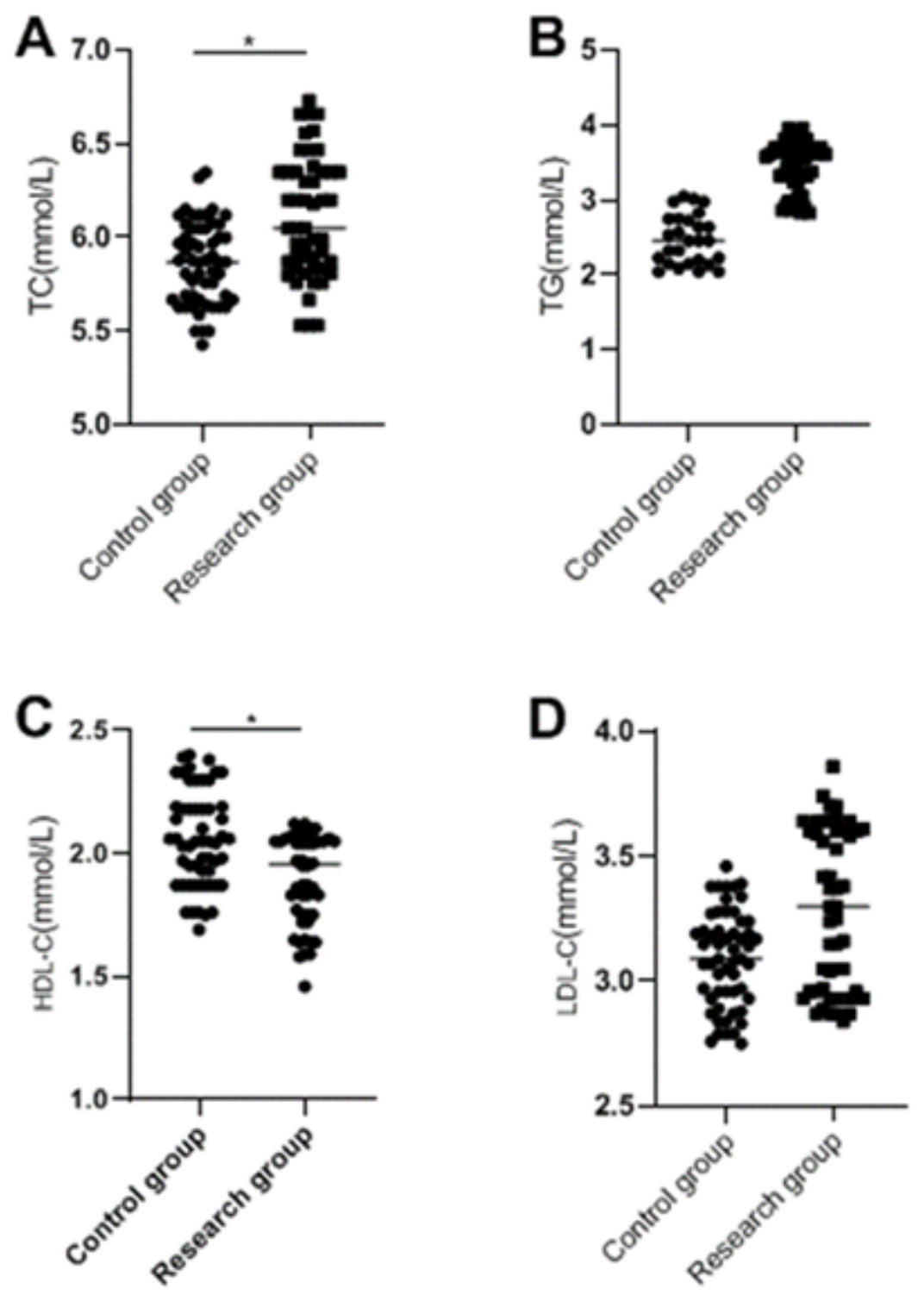

Figure 1

Analysis of blood lipid levels of pregnant women in the two groups After comparative analysis, A) TC level of pregnant women in the research group and control group, B) TG level of pregnant women in the research group and control group, C) HDL-C level of pregnant women in the research group and control group, and D) LDL-C level of pregnant women in the research group and control group. Data are expressed as mean $\pm S D$, where " $\star^{\prime \prime}$ is $(P<0.05)$. 


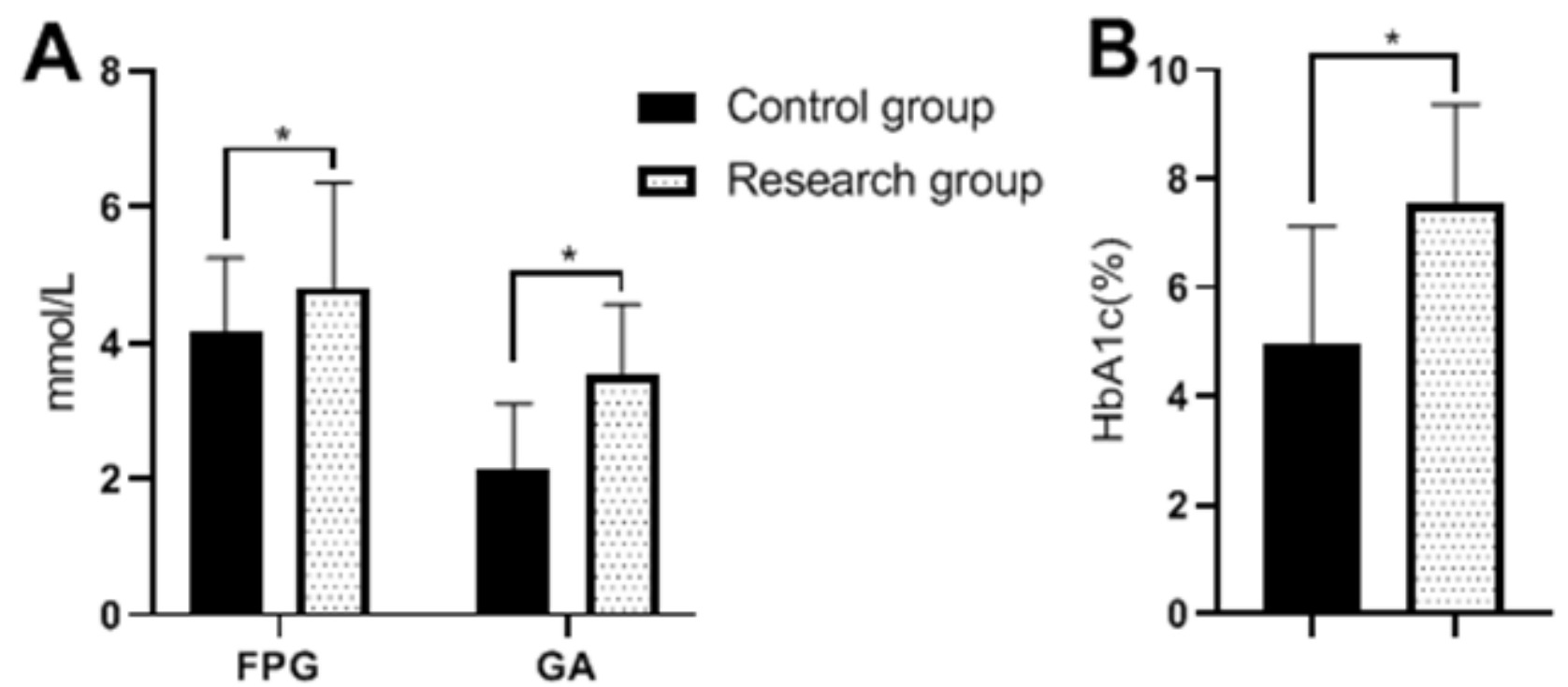

Figure 2

Analysis of the blood glucose levels of pregnant women in the two groups. A) FPG and GA levels of pregnant women in the research group and control group, and B) HbAlc level of pregnant women in the research group and the control group, Data are expressed as mean $\pm S D$, where " $*$ " is $(P<0.05)$.
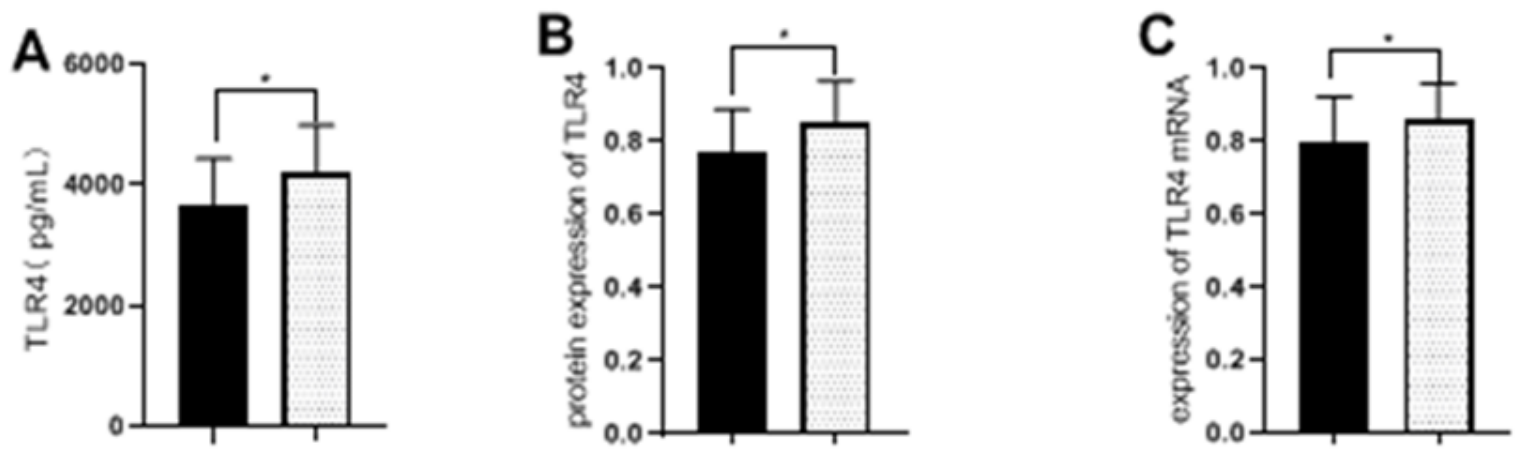

Figure 3

Analysis of the TLR4 levels of pregnant women in the two groups. A) the levels of TLR4 in the umbilical cord blood of the pregnant women in research group and control group, B) the relative expression of TLR4 protein in the placenta of the pregnant women in research group and control group, and C) TLR4 mRNA in the placenta in the research group and control group, Data are expressed as mean $\pm S D$, where “*” is $(P<0.05)$. 


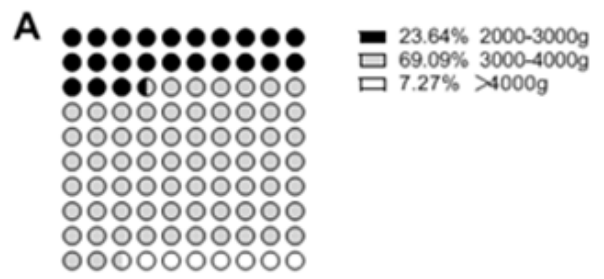

Total=55
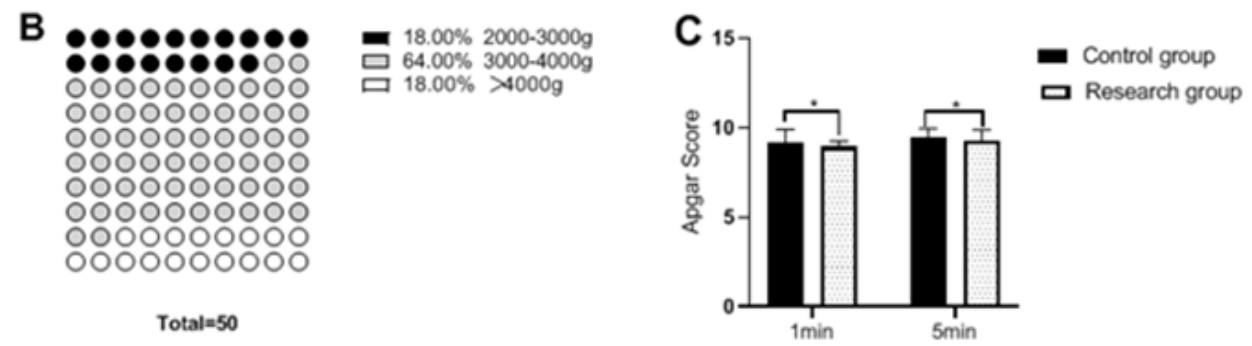

\section{Figure 4}

Analysis of the situation of newborns in the two groups. A) birth weight of newborns in the research group and B) control group. C) The scores of newborns at $1 \mathrm{~min}$ and $5 \mathrm{~min}$ in the research group were lower than those in the control group, Data are expressed as mean $\pm S D$, where " $\star$ " is $(P<0.05)$. 